\title{
Cerium oxide nanoparticles attenuate acute kidney injury induced by intra-abdominal infection in Sprague-Dawley rats
}

\author{
Nandini D. P. K. Manne ${ }^{1,2,3}$, Ravikumar Arvapalli ${ }^{1}$, Niraj Nepal ${ }^{1,4}$, Tolou Shokuhfar ${ }^{5}$, Kevin M. Rice ${ }^{1}$, \\ Shinichi Asano' and Eric R. Blough 1,3,4*
}

\begin{abstract}
Background: Intra-abdominal infection or peritonitis is a cause for great concern due to high mortality rates. The prognosis of severe intra-abdominal infection is significantly diminished in the presence of acute kidney injury (AKI) which is often characterized by renal tubular cell death that can lead to renal failure. The purpose of the current study is to examine the therapeutic efficacy of cerium oxide $\left(\mathrm{CeO}_{2}\right)$ nanoparticles for the treatment of peritonitis-induced AKI by polymicrobial insult.

Results: A one-time administration of $\mathrm{CeO}_{2}$ nanoparticles $(0.5 \mathrm{mg} / \mathrm{kg})$ in the absence of antibiotics or other supportive care, attenuated peritonitis-induced tubular dilatation and the loss of brush border in male Sprague-Dawley rats. These improvements in renal structure were accompanied by decreases in serum cystatin-C levels, reduced renal oxidative stress, diminished Stat-3 phosphorylation and an attenuation of caspase-3 cleavage suggesting that the nanoparticle treatment improved renal glomerular filtration rate, diminished renal inflammation and reduced renal apoptosis. Consistent with these data, further analysis demonstrated that the $\mathrm{CeO}_{2}$ nanoparticle treatment diminished peritonitis-induced increases in serum kidney injury molecule-1 (KIM-1), osteopontin, $\beta-2$ microglobulin and vascular endothelial growth factor-A (VEGF-A) levels. In addition, the nanoparticle attenuated peritonitis-induced hyperglycemia along with increases in blood urea nitrogen (BUN), serum potassium and sodium.

Conclusion: $\mathrm{CeO}_{2}$ nanoparticles scavenge reactive oxygen species and attenuate polymicrobial insult induced increase in inflammatory mediators and subsequent AKI. Taken together, the data indicate that $\mathrm{CeO}_{2}$ nanoparticles may be useful as an alternative therapeutic agent or in conjunction with standard medical care for the treatment of peritonitis induced acute kidney injury.
\end{abstract}

Keywords: Cerium oxide nanoparticles, Polymicrobial insult, Acute kidney injury, Reactive oxygen species, Peritonitis

\section{Background}

Intra-abdominal infection-induced peritonitis is characterized by an overwhelming immune response in reaction to the presence of infectious agents that can lead to multi-organ failure and death if not properly managed. Acute kidney injury (AKI) induced by polymicrobial insult is a cause of great concern as it increases mortality rates and is also associated with increased financial

\footnotetext{
*Correspondence: blough@marshall.edu

${ }^{1}$ Center for Diagnostic Nanosystems, Marshall University,

Huntington, WV, USA

Full list of author information is available at the end of the article
}

burden and length of hospital stay [1]. Despite advances in medical science and care, sepsis still baffles the research community due to its complex pathophysiology and rapid progression making current therapeutic interventions ineffective.

Intra-abdominal infection-induced acute renal failure is caused by a multitude of factors that includes components of ischemia-reperfusion injury, hypoperfusion, endothelial dysfunction, coagulation defects, cytokine storm and apoptosis [2-4]. Most studies to-date point that the single most cause for intra-abdominal infection induced AKI is the development of the systemic 
inflammatory response syndrome (SIRS) which is mediated by increased oxidative stress $[5,6]$. Other studies have shown that intra-abdominal infection causes injury to renal tubular cells by damaging the endothelium that leads to increased permeability [7]. These changes are histologically evident by loss of brush border and increased tubular dilatation [8]. At the cellular level, it is presumed that polymicrobial insult causes tubular cell death by caspase mediated apoptosis [2]. As such therapeutic interventions directed towards elimination of reactive oxygen species (ROS) may be warranted for the treatment of AKI.

Recent developments in nanotechnology have given rise to several promising compounds that can effectively scavenge ROS [9-11]. Among those, $\mathrm{CeO}_{2}$ nanoparticles have been increasingly used in biomedical field for the treatment of various pathologies involving oxidative stress $[12,13]$ and inflammation $[14,15]$. A property that is unique to $\mathrm{CeO}_{2}$ nanoparticles is the ability to cycle between $\mathrm{Ce}^{+3}$ (reduced) and $\mathrm{Ce}^{+4}$ (oxidized) states which allows them to effectively scavenge ROS [16]. Other studies have shown that $\mathrm{CeO}_{2}$ nanoparticles behave as a superoxide dismutase [17] and catalase [18] mimetic depending on the redox state of the particles. Researches have exploited this property of $\mathrm{CeO}_{2}$ nanoparticles for the treatment of diabetes $[19,20]$, cancer [21, 22] and ischemic stroke [23].

Previously we have shown that $\mathrm{CeO}_{2}$ nanoparticles inhibit intra-abdominal infection induced liver and heart dysfunction [24]. Whether $\mathrm{CeO}_{2}$ nanoparticles can be used to attenuate polymicrobial insult-induced AKI is not known. In continuation with our previous study, we evaluated the therapeutic efficacy of $\mathrm{CeO}_{2}$ nanoparticles for the treatment of polymicrobial insult-induced AKI by examining the effect of nanoparticle intervention on renal histological damage, oxidative stress, inflammation, apoptosis and changes in circulating AKI biomarkers.

\section{Results}

\section{Characterization of nanoceria}

Scanning transmission electron microscopy (STEM) was used to demonstrate the ordered structure of nanoparticles while transmission electron microscopy (TEM) demonstrated the size of $\mathrm{CeO}_{2}$ nanoparticles to be in between 10-40 nm (Fig. 1a, b). Energy dispersive X-ray spectroscopy (EDS) indicated that the content of cerium and oxygen in the sample to be 80.38 and $16.26 \%$, respectively (Fig. 1c).

\section{Cerium oxide nanoparticles attenuate peritonitis induced renal damage and breakdown of tubular F-actin}

Polymicrobial insult-induced AKI was characterized by renal tubular dilatation, loss of brush border and damage to the glomerular capillary network (Fig. 2c, g) which appeared to be attenuated with nanoparticle treatment (Fig. 2d, h). Renal sections of peritonitis induced animals showed marked loss of F-actin in proximal tubular cells (Fig. 3c) which was attenuated with nanoparticle treatment (Fig. 3d). The mean fluorescence intensity for F-actin was higher in peritonitis $+\mathrm{CeO}_{2}$ group by $61 \%$ when compared to peritonitis group alone (Fig. 3e) $(P<0.05)$.

\section{Cerium oxide nanoparticles attenuate peritonitis induced oxidative stress, stat-3 activation, cleavage of caspase 3 and serum biomarkers of renal failure}

Compared to control and treated animals, the renal sections obtained from animals suffering from peritonitis exhibited increased superoxide levels (Fig. 4c, e; $P<0.05$ ). Similarly, nanoparticle treatment decreased peritonitis induced increases in the ratio of phosphorylated to total levels of Stat-3 (Fig. 5a; $P<0.05$ ) and caspase-3 cleavage (Fig. 5b; $P<0.05$ ) at $18 \mathrm{~h}$. Compared to the control group and treated animals, peritonitis increased the levels of $\beta-2$ microglobulin at $3 \mathrm{~h}$ and $18 \mathrm{~h}$ and the levels of KIM1 , cystatin-C, osteopontin and VEGF-A at $18 \mathrm{~h}$ (Table 1; $P<0.05)$.

\section{Cerium oxide nanoparticles attenuate peritonitis induced changes in serum biochemical parameters}

Peritonitis caused a decrease in serum levels of sodium at $3 \mathrm{~h}$ and an increase in levels of potassium at $18 \mathrm{~h}$ (Table 2; $P<0.05$ ). Nanoparticle treatment attenuated peritonitis-induced changes in serum sodium and potassium (Table 2; $P<0.05$ ). Similarly, peritonitis induced early hyperglycemia and increases in levels of BUN at $3 \mathrm{~h}$ time point were attenuated with nanoparticles treatment (Table 2; $P<0.05$ ).

\section{Discussion}

Intra-abdominal infection or peritonitis-induced AKI has a poor prognosis despite recent advances in medical care. Studies have shown that peritonitis-induced AKI is associated with increases in oxidative and nitrosative stress which promote renal inflammation, tubular dilatation, vacuolization, sloughing of epithelial cells and loss of brush border which if severe can cause renal failure $[25,26] . \mathrm{CeO}_{2}$ nanoparticles have been shown to act as an anti-oxidant and anti-inflammatory agent in the treatment of several diseases including cancer and diabetes $[27,28]$. Similarly, previous work by our laboratory has showed that $\mathrm{CeO}_{2}$ nanoparticles can attenuate intra-abdominal infection-induced animal death along with liver and heart damage [24]. With this in mind, we hypothesized that $\mathrm{CeO}_{2}$ nanoparticles could be used to prevent peritonitis induced AKI. 

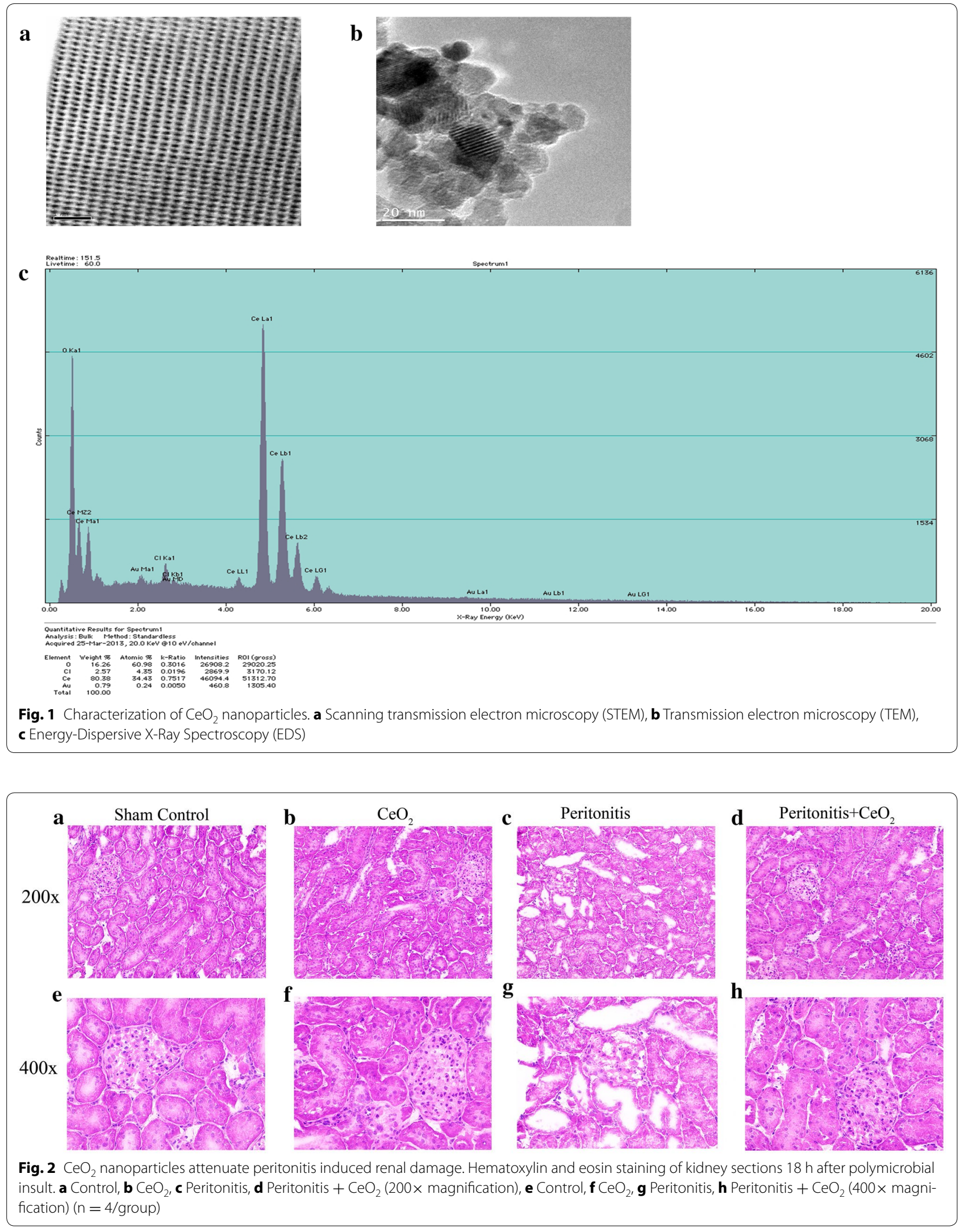


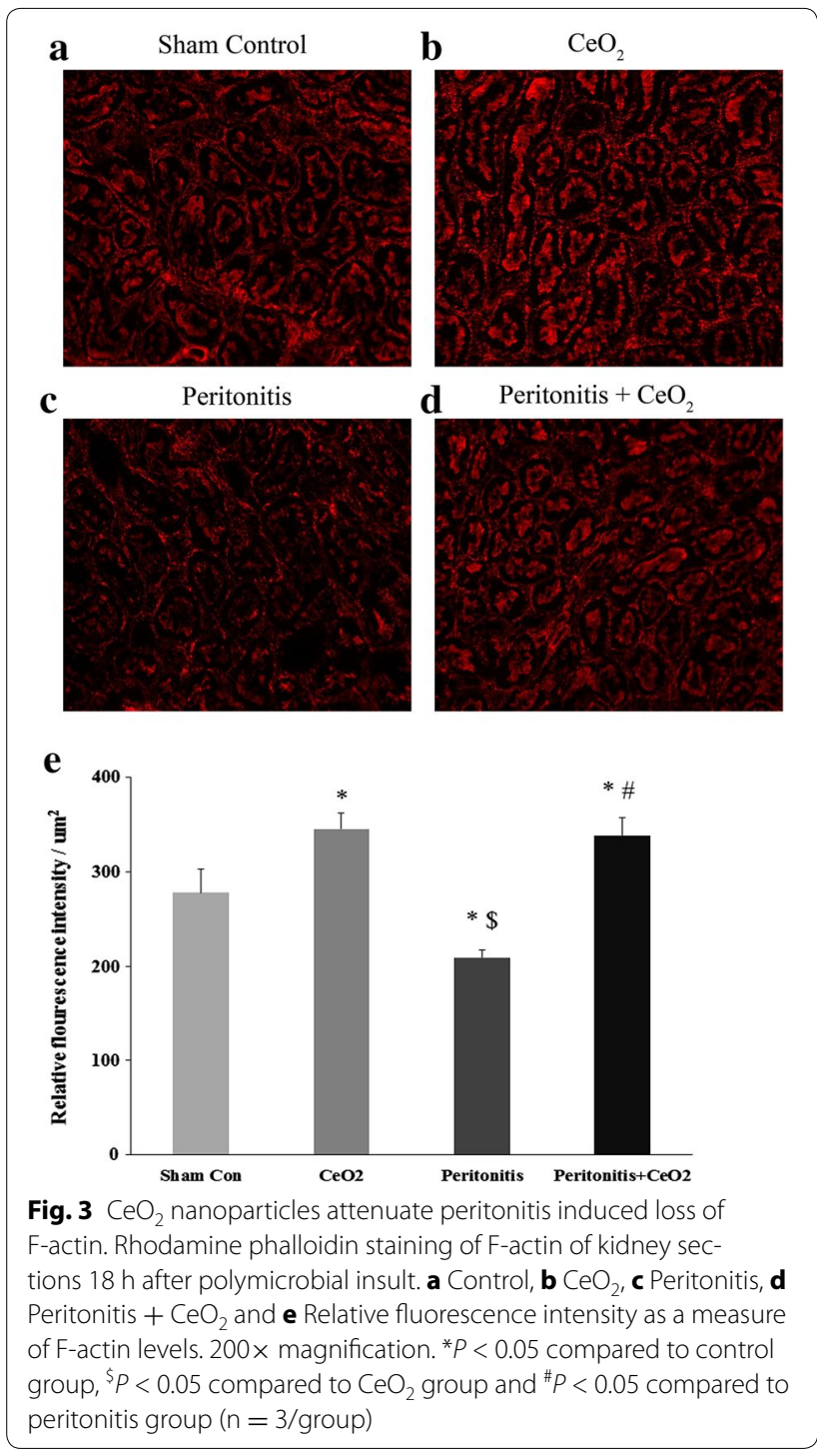

Previous studies have shown that peritonitis induced by polymicrobial insult leads to marked tubular damage along with loss of brush border and derangement in glomerular capillary network [29]. We found that the $\mathrm{CeO}_{2}$ nanoparticle treatment attenuated peritonitis-induced damage to the renal glomeruli and tubules (Fig. 1). AKI is characterized by the loss of F-actin that leads to disruption in cytoskeleton network and impairs renal structural and functional integrity [30]. To address this possibility, we next sought to determine whether the nanoparticles can prevent peritonitis-induced changes in renal structural integrity. As expected we found that peritonitis caused a significant decrease F-actin associated fluorescence which appeared to be attenuated with $\mathrm{CeO}_{2}$ nanoparticle treatment (Fig. 3). Taken together, these data suggest that the nanoparticle treatment was associated with diminished AKI.

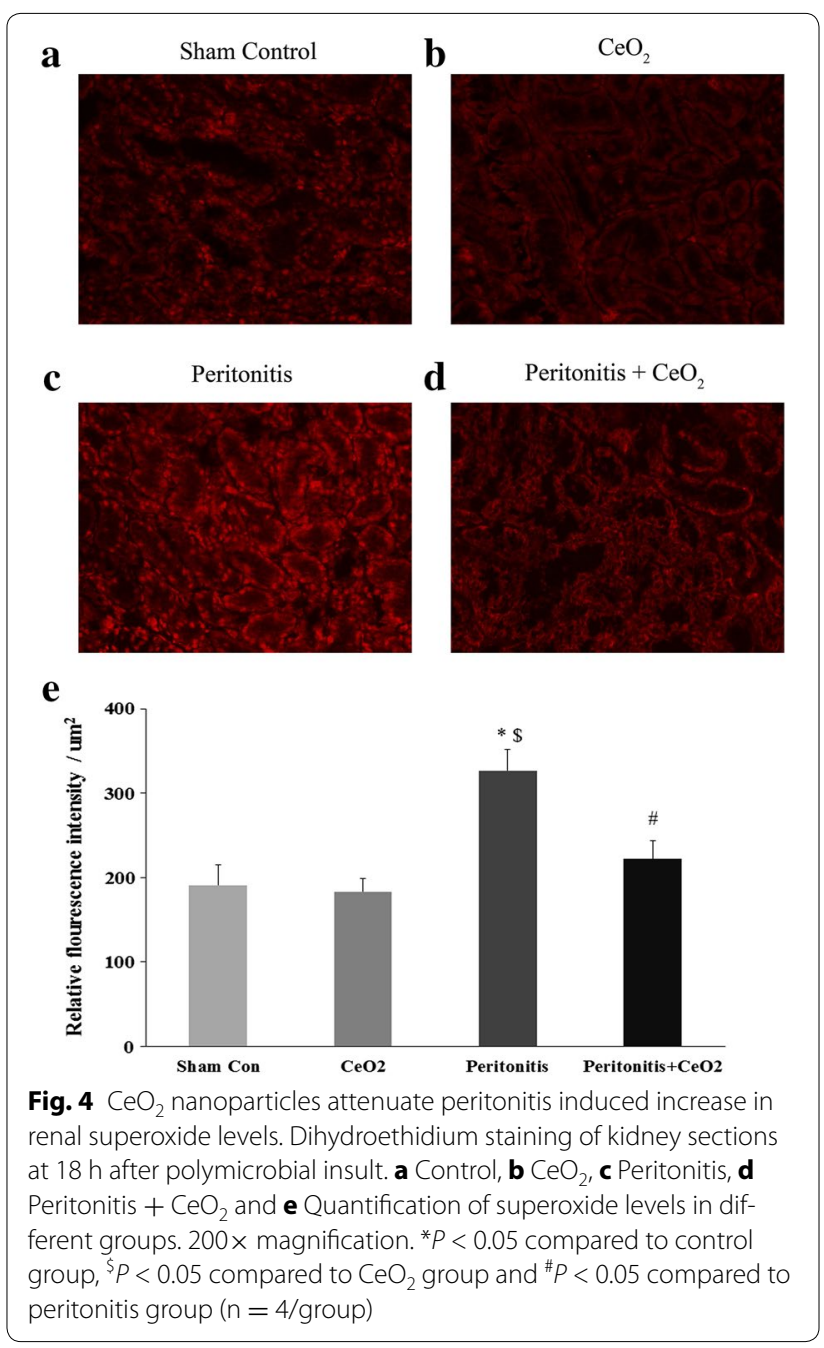

It is thought that peritonitis is characterized by increases in oxidative stress which can result in an uncontrolled systemic inflammatory response and multi-organ failure [31]. Studies have shown that $\mathrm{CeO}_{2}$ nanoparticles are potent ROS scavengers and that they can act as catalase and SOD mimetics [32]. Supporting these data, we found that $\mathrm{CeO}_{2}$ nanoparticle treatment significantly attenuated peritonitis induced-increases in renal superoxide oxide levels (Fig. 4). To extend these findings, we next examined the phosphorylation (activation) of the inflammatory mediated Jak-Stat proteins [33]. The Jak-Stat pathway has been shown to participate in the development of diabetic nephropathy, renal fibrosis and ischemia reperfusion injury where it functions as a key regulator of cytokine and growth factor signaling [34]. Additional data has also demonstrated that Stat-3 signaling is necessary for the hypoxia-induced transcription of VEGF [35]. Here we found that peritonitis-induced the activation of Stat-3 and increased serum VEGF levels. (Fig. 5a; Table 1). 


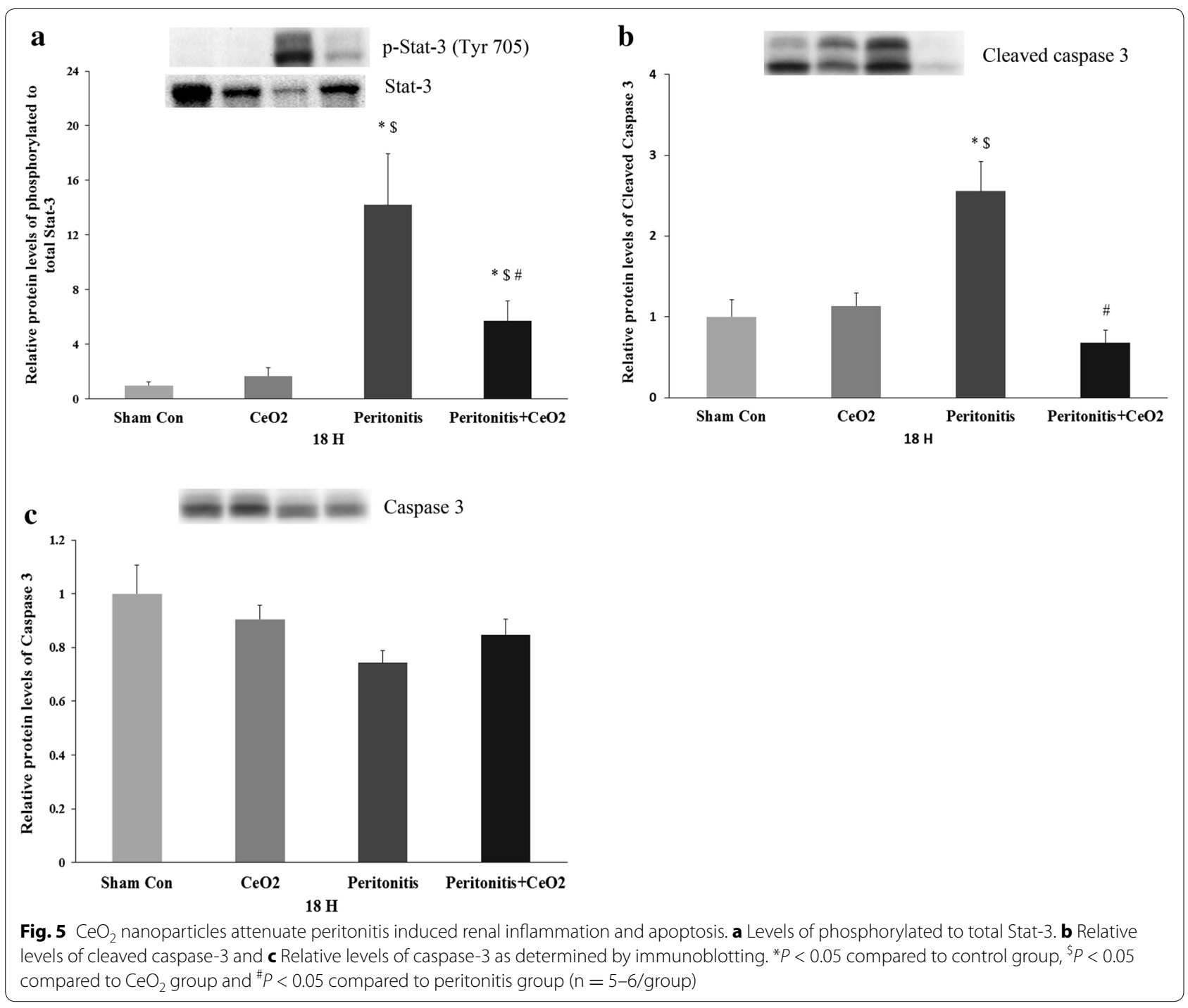

Similarly, a growing body of evidence suggests that the apoptosis of renal tubular cells is one of the major causes for AKI in peritonitis induced by polymicrobial insult [36]. Other work has demonstrated that the cleavage of caspase-3 activates gelsolin which has F-actin severing properties [37]. The loss of F-actin in turn, causes cytoskeletal derangement that can result in apoptotic cell death [30]. In agreement with our fluorescent measurement of actin levels, we found that peritonitis induced-cleavage of caspase-3 was significantly attenuated by $\mathrm{CeO}_{2}$ nanoparticle treatment (Figs. 3, 5a). Taken together and consistent with our histological findings of diminished renal damage, these data suggest that nanoparticle treatment is associated with decreased renal superoxide levels, reduced renal inflammation, and diminished renal apoptosis.
In addition to changes in cellular structure, AKI has also been shown to be associated with decreases in glomerular filtration rate and alterations in serum KIM-1, $\beta-2$ microglobulin, and osteopontin levels [38-40]. As expected, peritonitis was associated with alterations in the serum level of all of these variables which appeared to be significantly diminished with nanoparticle treatment (Table 1). In a similar fashion, hyponatremia, hyperkalemia, hyperglycemia, and increased blood urea nitrogen levels are generally found in acute renal failure with decreased renal tubular flow rate $[8,41]$. Consistent with these findings, we found similar changes in the present study, and importantly, demonstrated a reduction in these variables with nanoparticle treatment (Table 2). 


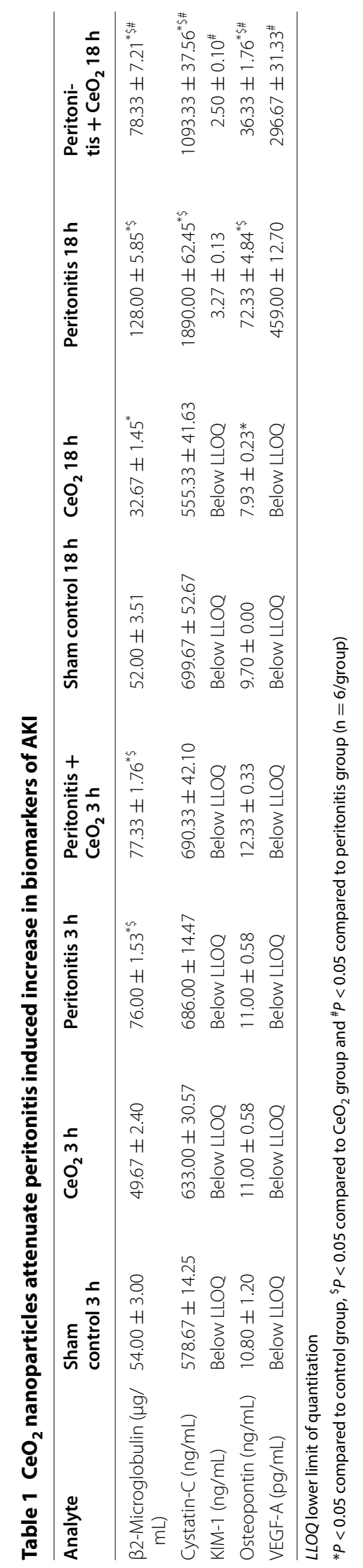




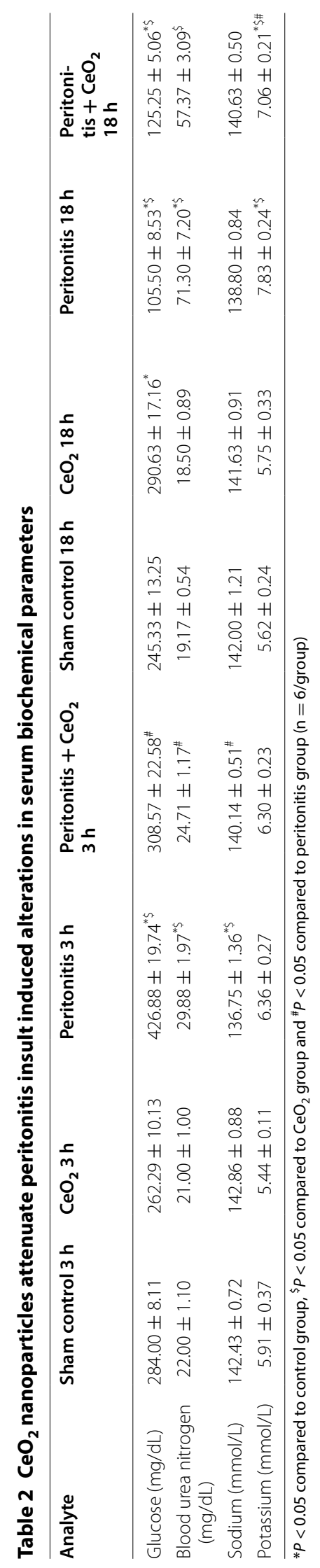




\section{Conclusion}

The results of the current study suggest that a single, "stand-alone" dose of $\mathrm{CeO}_{2}$ nanoparticles confers protection against severe polymicrobial insult-induced acute kidney injury. Our data suggest that the $\mathrm{CeO}_{2}$ nanoparticles act by scavenging reactive oxygen species which is associated with diminished caspase- 3 activation, reduced loss of $\mathrm{F}$-actin and less damage to the tubules. Improvements in kidney structure were accompanied by evidence of increased renal function and decreased serum biomarker levels of renal injury (Fig. 6). Taken together, these data suggest that further investigation into the potential utility of using $\mathrm{CeO}_{2}$ nanoparticles for the treatment of polymicrobial-induced AKI may be warranted.

\section{Methods}

\section{Characterization of $\mathrm{CeO}_{2}$ nanoparticles}

$\mathrm{CeO}_{2}$ nanoparticles were commercially purchased from US Research Nanomaterials Inc. (Houston, TX, USA). Scanning transmission electron microscopy (STEM) images were obtained by using Aberration Corrected Analytical Electron Microscope (TEM/STEM JEOL
JEM-ARM200CF, Japan) operated at $200 \mathrm{keV}$. Particle size was obtained using JEOL JEM-2010 transmission electron microscope (TEM). Sample purity was estimated by energy dispersive $\mathrm{X}$-ray spectroscopy using Noran Voyager EDX software.

\section{Polymicrobial insult and $\mathrm{CeO}_{2}$ nanoparticle treatment}

Male Sprague-Dawley rats aged 10 weeks were purchased from Hill-Top laboratories and allowed to acclimatize for 2 weeks prior to experimentation. All surgical procedures were performed in accordance with the guidelines provided by Marshall University Institutional Animal Care and Use Committee (IACUC), and Association for Assessment and Accreditation of Laboratory Animal Care (AAALAC). Briefly, animals were anesthetized under isoflurane and a small mid ventral incision of $0.5 \mathrm{~cm}$ was made. Sham controls and $\mathrm{CeO}_{2}$ only groups were injected with $5 \mathrm{ml} / \mathrm{kg}$ of $5 \%$ sterile dextrose solution intra-peritoneally (i.p) while peritonitis and peritonitis $+\mathrm{CeO}_{2}$ groups received cecal inoculum of $600 \mathrm{mg} /$ $\mathrm{kg} \mathrm{BW}$ in $5 \mathrm{ml} / \mathrm{kg} \mathrm{BW}$ of $5 \%$ sterile dextrose solution i.p as described previously [42]. Cecal material was obtained

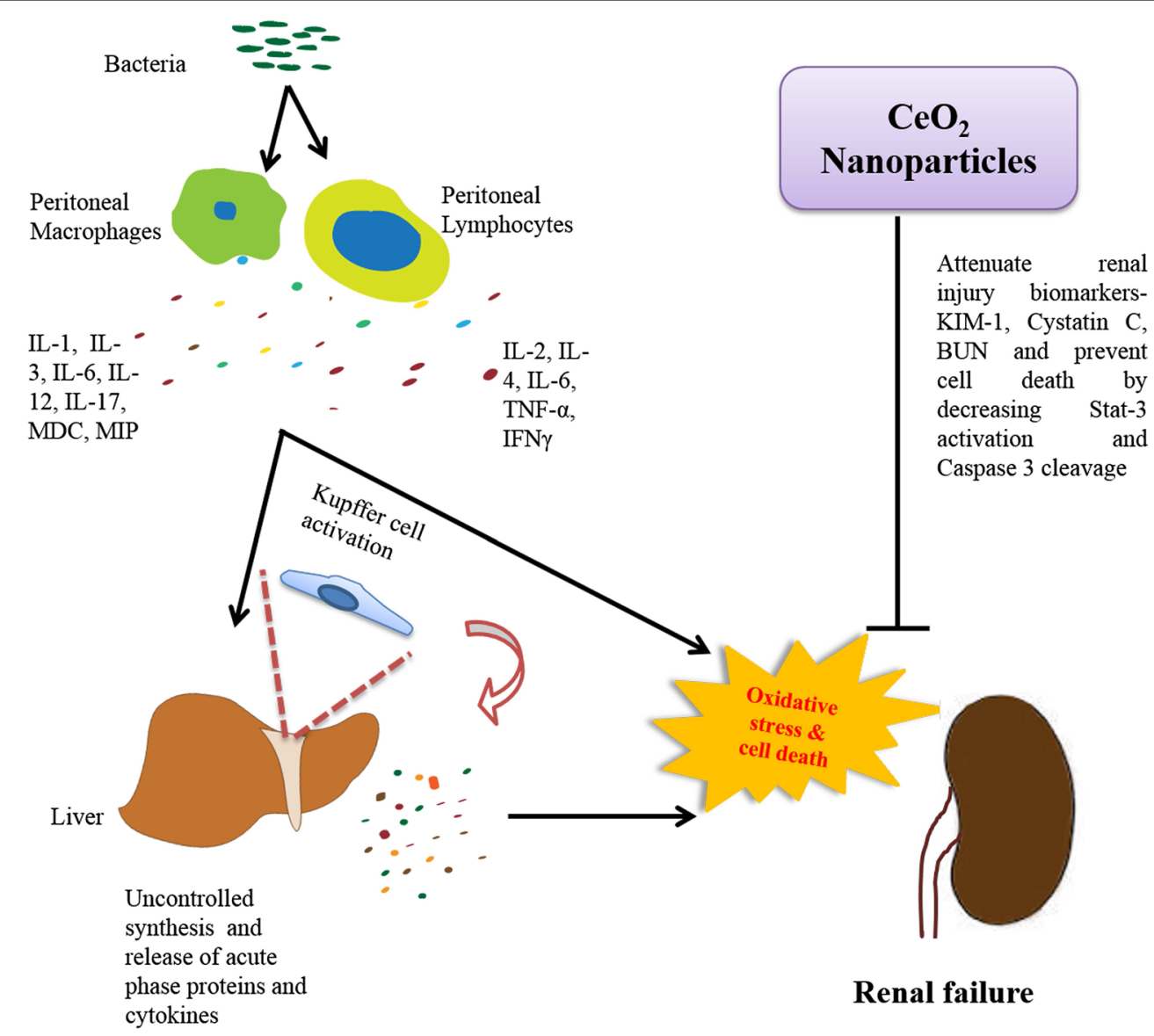

Fig. 6 Schematic representation of mechanism of action of $\mathrm{CeO}_{2}$ nanoparticles on polymicrobial insult induced AKI 
from healthy donor rats. The sham control and peritonitis groups were injected with $200 \mu \mathrm{l}$ of sterile distilled water intravenously while the $\mathrm{CeO}_{2}$ and peritonitis $+\mathrm{CeO}_{2}$ groups received $\mathrm{CeO}_{2}$ nanoparticles $(0.5 \mathrm{mg} / \mathrm{kg})$ in $200 \mu \mathrm{l}$ of sterile distilled water via tail vein injection.

\section{Tissue collection}

Animals were humanely sacrificed under anesthesia and the kidneys were excised, capsule removed and washed in Krebs-Ringer bicarbonate buffer (KRB) to remove any blood. Kidneys were frozen in liquid nitrogen and stored at $-80{ }^{\circ} \mathrm{C}$ until further analysis. Serum was obtained from whole blood by centrifugation at $5000 \times g$ for $10 \mathrm{~min}$ at room temperature and stored at $-80^{\circ} \mathrm{C}$.

\section{Renal histology and staining for F-actin}

Frozen kidneys were sectioned $(4 \mu \mathrm{m})$ with a Leica CM1950 cryostat and transferred to poly-L-lysine coated slides. Hematoxylin and eosin staining was performed using Histoperfect kit (BBC Biochemical, Seattle WA, USA) to assess kidney morphology and imaged using Evos XL microscope (Life Technologies, Grand Island, NY, USA).

Renal sections were stained for F-actin using rhodamine phalloidin (Life technologies, Grand Island, NY, USA). Briefly, frozen sections were washed with PBS and fixed in $4 \%$ methanol free formaldehyde for $10 \mathrm{~min}$ and washed with PBS $(3 \times 5 \mathrm{~min})$. Sections were then permeabilized with $0.3 \%$ Triton X-100 in PBS for 20 min and washed with PBS $(3 \times 5 \mathrm{~min})$. After blocking with $1 \%$ BSA for $30 \mathrm{~min}$, the tissue sections were incubated with $0.165 \mu \mathrm{M}$ rhodamine phalloidin for $20 \mathrm{~min}$ in the dark, washed with PBS $(3 \times 5 \mathrm{~min})$, cover slipped and then imaged with a Evos FL microscope (Life Technologies, Grand Island, NY, USA). Four images per section from at least three different animals from each group were evaluated for fluorescence intensity using Image $\mathrm{J}$ analysis software.

\section{Estimation of renal superoxide levels}

Superoxide levels in renal sections were estimated using dihydroethidium staining as described previously [43]. Briefly, sections were washed with PBS and incubated with $5 \mathrm{mM}$ dihydroethidium for $1 \mathrm{~h}$ at room temperature in the dark. After washing with PBS $(3 \times 5 \mathrm{~min})$, sections were imaged with an Evos FL microscope (Life Technologies, Grand Island, NY, USA). Four images per section from at least four different animals from each group were evaluated for fluorescence intensity using Image J analysis software.

\section{SDS-PAGE and immunoblotting}

Approximately $100 \mathrm{mg}$ of frozen kidney was taken and pulverized to a fine powder, suspended in $900 \mu \mathrm{l}$ of T-PER
(Pierce, Rockford, IL, USA) containing $1 \%$ protease and phosphatase inhibitors and homogenized. Supernants were collected by centrifugation $(10,000 \times g$ for $10 \mathrm{~min}$ at $4{ }^{\circ} \mathrm{C}$ and the protein concentration determined using the $660 \mathrm{~nm}$ assay (Pierce, Rockford, IL, USA). Samples were equally diluted with $4 \times$ Laemilli buffer and then subjected to electrophoresis on $10 \%$ PAGEr Gold Precast gel (Lonza, Rockland, ME, USA) before transfer to nitrocellulose membranes as detailed elsewhere [44]. Equal loading of protein was verified by Ponceau $\mathrm{S}$ staining of nitrocellulose membranes. Membranes were blocked with $5 \%$ milk in TBST for $1 \mathrm{~h}$ and later probed with primary antibodies against p-stat-3 (Tyr 705), stat-3, cleaved caspase-3 and caspase-3 (Cell Signaling Technology, Danvers, MA, USA). After exposure to the primary antibodies, membranes were washed with TBST $(3 \times 5 \mathrm{~min})$ and incubated with secondary anti-rabbit (Cell Signaling Technology, Danvers, MA, USA) for $1 \mathrm{~h}$ at room temperature. Immunoreactivity was visualized using Supersignal West Pico Chemiluminiscent substrate (Pierce, Rockford, IL, USA) and quantified by Fluorchem 9900 software (Protein Simple, Santa Clara, CA, USA).

\section{Multiplex immunoassay and serum biochemical analysis}

Serum samples from different animals in each group were pooled and sent to Myriad RBM (Austin, TX, USA) for the analysis of KIM-1, cystatin-C, osteopontin, $\beta-2$ microglobulin and VEGF-A using the rodent kidney MAP. Samples were run in triplicate for statistical analysis. The amount of glucose, BUN, sodium and potassium were determined in serum using an Abaxis VetScan ${ }^{\circledR}$ analyzer (Abaxis, Union City, CA, USA).

\section{Statistical analysis}

Results are presented in the form of mean \pm standard error of mean. Differences in dependent variables across groups were determined by one way analysis of variance (ANOVA) with Student Newman Keul's post hoc analysis or ANOVA on ranks with Student Newman Keul's post hoc analysis for non-normally distributed samples. Where appropriate a $t$ test was used. A probability value of $P<0.05$ was accepted to be statistically significant.

\section{Authors' contributions}

ERB designed and supervised the study, contributed to writing the article and finalization of article. NDPM designed the study and performed all the experiments involved, analyzed data and drafted the manuscript. RA, NN, TS, KMR, SA conducted experiments and analyzed the data. All the authors read and approved the manuscript.

\section{Author details}

${ }^{1}$ Center for Diagnostic Nanosystems, Marshall University, Huntington, WV, USA. ${ }^{2}$ Department of Public Health, Marshall University, Huntington, WV USA. ${ }^{3}$ Department of Pharmaceutical Sciences and Research, Marshall University, Huntington, WV, USA. ${ }^{4}$ Department of Pharmacology, Physiology 
and Toxicology, Marshall University, Huntington, WV, USA. ${ }^{5}$ Department of Mechanical Engineering and Engineering Mechanics, Michigan Technological University, Houghton, MI, USA

\section{Acknowledgements}

This work was supported in part from DOE grant (DE-PS02-09ER-01 to E.R.B).

\section{Competing interests}

The authors declare that they have no competing interests.

Received: 23 July 2015 Accepted: 13 October 2015

Published online: 24 October 2015

\section{References}

1. Majumdar A. Sepsis-induced acute kidney injury. Indian J Crit Care Med Peer-Review Off Publ Indian Soc Crit Care Med. 2010;14(1):14-21. doi:10.4103/0972-5229.63031.

2. Lee SY, Lee YS, Choi HM, Ko YS, Lee HY, Jo SK, et al. Distinct pathophysiologic mechanisms of septic acute kidney injury: role of immune suppression and renal tubular cell apoptosis in murine model of septic acute kidney injury. Crit Care Med. 2012;40(11):2997-3006. doi:10.1097/ CCM.0b013e31825b912d.

3. Bougle A, Duranteau J. Pathophysiology of sepsis-induced acute kidney injury: the role of global renal blood flow and renal vascular resistance. Contrib Nephrol. 2011;174:89-97. doi:10.1159/000329243.

4. StoyanoffTR, Todaro JS, Aguirre MV, Zimmermann MC, Brandan NC. Amelioration of lipopolysaccharide-induced acute kidney injury by erythropoietin: involvement of mitochondria-regulated apoptosis. Toxicology. 2014;318:13-21. doi:10.1016/j.tox.2014.01.011.

5. Schrier RW, Wang W. Acute renal failure and sepsis. N Engl J Med. 2004;351(2):159-69. doi:10.1056/NEJMra032401.

6. Wang Z, Holthoff JH, Seely KA, Pathak E, Spencer HJ 3rd, Gokden N, et al. Development of oxidative stress in the peritubular capillary microenvironment mediates sepsis-induced renal microcirculatory failure and acute kidney injury. Am J Pathol. 2012;180(2):505-16. doi:10.1016/j. ajpath.2011.10.011.

7. Xu C, Chang A, Hack BK, Eadon MT, Alper SL, Cunningham PN. TNF-mediated damage to glomerular endothelium is an important determinant of acute kidney injury in sepsis. Kidney Int. 2014;85(1):72-81. doi:10.1038/ ki.2013.286.

8. Doi K, Leelahavanichkul A, Yuen PS, Star RA. Animal models of sepsis and sepsis-induced kidney injury. J Clin Invest. 2009;119(10):2868-78. doi:10.1172/JCl39421.

9. Katsumi H, Fukui K, Sato K, Maruyama S, Yamashita S, Mizumoto E, et al. Pharmacokinetics and preventive effects of platinum nanoparticles as reactive oxygen species scavengers on hepatic ischemia/reperfusion injury in mice. Metallomics Integr Biometal Sci. 2014;6(5):1050-6. doi:10.1039/c4mt00018h.

10. Yang $X$, Jin L, Yao L, Shen FH, Shimer AL, Li X. Antioxidative nanofullerol prevents intervertebral disk degeneration. Int J Nanomed. 2014;9:241930. doi:10.2147/IJN.S60853.

11. Chistyakov VA, Smirnova YO, Prazdnova EV, Soldatov AV. Possible mechanisms of fullerene C(6)(0) antioxidant action. BioMed Res Int. 2013;2013:821498. doi:10.1155/2013/821498.

12. Chen S, Hou Y, Cheng G, Zhang C, Wang S, Zhang J. Cerium oxide nanoparticles protect endothelial cells from apoptosis induced by oxidative stress. Biol Trace Elem Res. 2013;154(1):156-66. doi:10.1007/ s12011-013-9678-8

13. Kolli MB, Manne ND, Para R, Nalabotu SK, Nandyala G, Shokuhfar T, et al. Cerium oxide nanoparticles attenuate monocrotaline induced right ventricular hypertrophy following pulmonary arterial hypertension. Biomaterials. 2014;35(37):9951-62. doi:10.1016/j.biomaterials.2014.08.037.

14. Kyosseva SV, Chen L, Seal S, McGinnis JF. Nanoceria inhibit expression of genes associated with inflammation and angiogenesis in the retina of VIdlr null mice. Exp Eye Res. 2013;116:63-74. doi:10.1016/j. exer.2013.08.003.
15. Chaudhury K, Babu KN, Singh AK, Das S, Kumar A, Seal S. Mitigation of endometriosis using regenerative cerium oxide nanoparticles. Nanomed Nanotechnol Biol Med. 2013;9(3):439-48. doi:10.1016/j.nano.2012.08.001.

16. Lee SS, Song W, Cho M, Puppala HL, Nguyen P, Zhu H, et al. Antioxidant properties of cerium oxide nanocrystals as a function of nanocrystal diameter and surface coating. ACS Nano. 2013;7(11):9693-703. doi:10.1021/nn4026806.

17. Clark A, Zhu A, Sun K, Petty HR. Cerium oxide and platinum nanoparticles protect cells from oxidant-mediated apoptosis. J Nanoparticle Res Interdiscipl Forum Nanoscale Sci Technol. 2011;13(10):5547-55. doi:10.1007/ s11051-011-0544-3.

18. Pirmohamed T, Dowding JM, Singh S, Wasserman B, Heckert E, Karakoti AS, et al. Nanoceria exhibit redox state-dependent catalase mimetic activity. Chem Commun. 2010;46(16):2736-8. doi:10.1039/b922024k.

19. Pourkhalili N, Hosseini A, Nili-Ahmadabadi A, Hassani S, Pakzad M, Baeeri $M$, et al. Biochemical and cellular evidence of the benefit of a combination of cerium oxide nanoparticles and selenium to diabetic rats. World J Diabetes. 2011;2(11):204-10. doi:10.4239/wjd.v2.i11.204.

20. Hosseini A, Baeeri M, Rahimifard M, Navaei-Nigjeh M, Mohammadirad A, Pourkhalili N, et al. Antiapoptotic effects of cerium oxide and yttrium oxide nanoparticles in isolated rat pancreatic islets. Hum Exp Toxicol. 2013:32(5):544-53. doi:10.1177/0960327112468175.

21. Gao Y, Chen K, Ma JL, Gao F. Cerium oxide nanoparticles in cancer. OncoTargets Therapy. 2014;7:835-40. doi:10.2147/OTT.S62057.

22. Alili L, Sack M, von Montfort C, Giri S, Das S, Carroll KS, et al. Downregulation of tumor growth and invasion by redox-active nanoparticles. Antioxid Redox Signal. 2013;19(8):765-78. doi:10.1089/ars.2012.4831.

23. Estevez AY, Pritchard S, Harper K, Aston JW, Lynch A, Lucky JJ, et al. Neuroprotective mechanisms of cerium oxide nanoparticles in a mouse hippocampal brain slice model of ischemia. Free Radic Biol Med. 2011;51(6):1155-63. doi:10.1016/j.freeradbiomed.2011.06.006.

24. Manne ND, Arvapalli R, Nepal N, Thulluri S, Selvaraj V, Shokuhfar T, et al. Therapeutic potential of cerium oxide nanoparticles for the treatment of peritonitis induced by polymicrobial insult in Sprague-Dawley rats. Crit Care Med. 2015; doi:10.1097/CCM.0000000000001258.

25. Pinto CF, Watanabe M, da Fonseca CD, Ogata Cl, Vattimo Mde F. The sepsis as cause of acute kidney injury: an experimental model. Revista da Escola de Enfermagem da U S P. 2012;46: 86-90.

26. Wu L, Gokden N, Mayeux PR. Evidence for the role of reactive nitrogen species in polymicrobial sepsis-induced renal peritubular capillary dysfunction and tubular injury. J Am Soc Nephrol JASN. 2007;18(6):1807-15. doi:10.1681/ASN.2006121402.

27. Sack M, Alili L, Karaman E, Das S, Gupta A, Seal S, et al. Combination of conventional chemotherapeutics with redox-active cerium oxide nanoparticles-a novel aspect in cancer therapy. Mol Cancer Ther. 2014;. doi:10.1158/1535-7163.MCT-13-0950.

28. Younce CW, Wang K, Kolattukudy PE. Hyperglycaemia-induced cardiomyocyte death is mediated via MCP-1 production and induction of a novel zinc-finger protein MCPIP. Cardiovasc Res. 2010;87(4):665-74. doi:10.1093/cvr/cvq102

29. Adembri C, Sgambati E, Vitali L, Selmi V, Margheri M, Tani A, et al. Sepsis induces albuminuria and alterations in the glomerular filtration barrier: a morphofunctional study in the rat. Crit Care. 2011;15(6):R277. doi:10.1186/cc10559.

30. Park SW, Chen SW, Kim M, D'Agati VD, Lee HT. Human heat shock protein 27-overexpressing mice are protected against acute kidney injury after hepatic ischemia and reperfusion. Am J Physiol Renal Physiol. 2009;297(4):F885-94. doi:10.1152/ajprenal.00317.2009.

31. Bosmann M, Ward PA. The inflammatory response in sepsis. Trends Immunol. 2013;34(3):129-36. doi:10.1016/j.it.2012.09.004.

32. Hirst SM, Karakoti A, Singh S, SelfW, Tyler R, Seal S, et al. Bio-distribution and in vivo antioxidant effects of cerium oxide nanoparticles in mice. Environ Toxicol. 2013;28(2):107-18. doi:10.1002/tox.20704.

33. Yang $N$, Luo $M$, Li R, Huang $Y$, Zhang $R$, Wu Q, et al. Blockage of JAK/STAT signalling attenuates renal ischaemia-reperfusion injury in rat. Nephrol Dial Transplant Off Publ Eur Dial Transplant Assoc Eur Renal Assoc. 2008;23(1):91-100. doi:10.1093/ndt/gfm509.

34. Rawlings JS, Rosler KM, Harrison DA. The JAK/STAT signaling pathway. J Cell Sci. 2004;117(Pt 8):1281-3. doi:10.1242/jcs.00963. 
35. Hoeben A, Landuyt B, Highley MS, Wildiers H, Van Oosterom AT, De Bruijn EA. Vascular endothelial growth factor and angiogenesis. Pharmacol Rev. 2004;56(4):549-80. doi:10.1124/pr.56.4.3.

36. Kockara A, Kayatas M. Renal cell apoptosis and new treatment options in sepsis-induced acute kidney injury. Ren Fail. 2013;35(2):291-4. doi:10.310 9/0886022X.2012.744040.

37. Kothakota S, Azuma T, Reinhard C, Klippel A, Tang J, Chu K, et al. Caspase3-generated fragment of gelsolin: effector of morphological change in apoptosis. Science. 1997;278(5336):294-8.

38. Doi K, Yuen PS, Eisner C, Hu X, Leelahavanichkul A, Schnermann J, et al. Reduced production of creatinine limits its use as marker of kidney injury in sepsis. J Am Soc Nephrol JASN. 2009;20(6):1217-21.

39. Vaidya VS, Ferguson MA, Bonventre JV. Biomarkers of acute kidney injury. Annu Rev Pharmacol Toxicol. 2008;48:463-93. doi:10.1146/annurev. pharmtox.48.113006.094615.

40. Zhang Z. Biomarkers, diagnosis and management of sepsisinduced acute kidney injury: a narrative review. Heart Lung Vessels. 2015;7(1):64-73
41. Hannon RJ, Boston VE. Hyponatraemia and intracellular water in sepsis: an experimental comparison of the effect of fluid replacement with either $0.9 \%$ saline or $5 \%$ dextrose. J Pediatr Surg. 1990;25(4):422-5.

42. Chopra M, Golden HB, Mullapudi S, Dowhan W, Dostal DE, Sharma AC. Modulation of myocardial mitochondrial mechanisms during severe polymicrobial sepsis in the rat. PLoS One. 2011;6(6):e21285. doi:10.1371/ journal.pone.0021285.

43. Wang C, Blough ER, Arvapalli R, Dai X, Paturi S, Manne N, et al. Metabolic syndrome-induced tubulointerstitial injury: role of oxidative stress and preventive effects of acetaminophen. Free Radic Biol Med. 2013;65:141726. doi:10.1016/j.freeradbiomed.2013.10.005.

44. Manne ND, Lima M, Enos RT, Wehner P, Carson JA, Blough E. Altered cardiac muscle $\mathrm{mTOR}$ regulation during the progression of cancer cachexia in the ApcMin/+mouse. Int J Oncol. 2013;42(6):2134-40. doi:10.3892/ ijo.2013.1893.

\section{Submit your next manuscript to BioMed Central and take full advantage of:}

- Convenient online submission

- Thorough peer review

- No space constraints or color figure charges

- Immediate publication on acceptance

- Inclusion in PubMed, CAS, Scopus and Google Scholar

- Research which is freely available for redistribution

Submit your manuscript at

www.biomedcentral.com/submit

C Biomed Central 